\title{
MANAGEMENT OF STRABISMUS DUE TO ORBITAL MYOSITIS
}

\author{
D. A. R. BESSANT and J. P. LEE \\ London
}

\begin{abstract}
SUMMARY
We report on 5 consecutive patients seen at the botulinum toxin clinic at Moorfields Eye Hospital with an ocular motility disorder secondary to orbital myositis. CT scans demonstrated involvement of one or both of the medial recti in the inflammatory process in all 5 patients. In addition 1 patient had involvement of both the lateral recti and the right superior rectus. Two patients had been treated with oral steroids, 3 with nonsteroidal anti-inflammatory agents, and 1 with orbital radiotherapy. Prior to toxin injection 3 patients had an esotropia (ranging from $4 \Delta$ to $30 \Delta$ ) and two an exotropia (52 $\Delta$ and $85 \Delta$ ). A vertical imbalance was present in 3 , and all 5 patients had symptomatic diplopia. A total of six injections were given to 5 patients, 2 of whom later went on to have surgery. Toxin injection reduced the angle of the deviation to less than $10 \Delta$ in 4 patients, all of whom are now asymptomatic. The fifth patient has persistent diplopia despite two operations to correct a large exotropia. We discuss the role of botulinum toxin and surgery in the management of strabismus due to orbital myositis.
\end{abstract}

Orbital myositis has been defined as a sub-group of the non-specific orbital inflammatory (pseudotumour) syndromes in which one or more of the extraocular muscles are primarily infiltrated by an inflammatory process. ${ }^{1}$ It may be acute, sub-acute or recurrent. Patients suffering from an acute episode of orbital myositis typically present with acute orbital pain, chemosis and conjunctival injection over the affected extraocular muscles, and a resultant ophthalmoplegia with diplopia. ${ }^{2,3}$ Ptosis, lid swelling and proptosis may also be seen.

Blodi and $\mathrm{Gass}^{4}$ in 1968 recorded an ocular motility disorder in 40 of their 140 patients with a diagnosis of non-specific orbital inflammation (pseudotumour), but classified only 6 as suffering from orbital myositis. Their classification was based

Correspondence to: D. A. R. Bessant, Moorfields Eye Hospital, City Road, London EC1V 2PD, UK. on biopsy specimens and they suggested that inflammatory infiltration of the extraocular muscles would be found more often if surgeons chose to biopsy the muscles more frequently in patients with 'pseudotumour'. The advent of CT and MRI scans has greatly improved the clinician's ability to identify which orbital tissues are primarily affected by an inflammatory process. ${ }^{5,6}$ The recent publication of several papers on orbital myositis suggests that this diagnosis is being made more frequently.,3,7-11

In a clinical setting orbital myositis must also be distinguished from dysthyroid eye disease (TED) which may also produce a bilateral asymmetric ophthalmoplegia. An acute onset with pain, which may increase on eye movement, is typical of myositis, whilst TED tends to have a more insidious onset with only superficial irritation. ${ }^{12}$ Lid retraction and lid lag are highly suggestive of TED, whilst a ptosis may occur in myositis. On a CT scan enlargement of the extraocular muscle which extends anteriorly to involve the tendon has been described in orbital myositis in contrast to the fusiform swelling which is usually seen in TED. ${ }^{5}$ Thyroid function tests are usually, though not always, abnormal in TED. Finally there is usually an excellent and rapid response to high-dose oral steroids in the acute phase of orbital myositis, whilst the response is slower and more variable in TED.

Whilst there have been several studies of both the clinical features and the histopathology of nonspecific orbital inflammation and myositis none of these has considered the management of the ocular motility defects that may persist after an episode of inflammation has settled down. ${ }^{1-4,7-13}$ In the one series in which this information was recorded, 6 of the 12 patients with orbital myositis had 'a persistent motility defect at the final examination', though the nature of these defects was not specified. ${ }^{3}$ In this study we describe how 5 patients with persistent diplopia after an episode of orbital myositis were managed, utilising botulinum toxin $\mathrm{A}$ injections and 
adjustable strabismus surgery, in the Strabismus Service at Moorfields Eye Hospital.

\section{PATIENTS AND METHODS}

Our subjects were 5 consecutive patients, presenting initially to the Orbital Clinic at Moorfields Eye Hospital, who had each suffered one or more episodes of orbital myositis resulting in a persistent ocular motility defect associated with significant diplopia. In all cases the diagnosis of orbital myositis was based upon a history of painful ophthalmoplegia, a thorough clinical examination, and a CT scan showing enlargement of one or more of the extraocular muscles. Dysthyroid eye disease (TED) and underlying systemic inflammatory disease were excluded by careful history taking, the absence of signs of TED on clinical examination, and appropriate laboratory investigations where indicated (thyroid function tests and an immunological screen including immunoglobulin IgG, IgM and IgA levels, and specific anti-thyroid and anti-nuclear antibodies).

All 5 patients were initially treated with an injection of botulinum toxin A (BTXA). This may be used both as a diagnostic tool, enabling the surgeon to determine whether a patient has the potential to regain binocular single vision after surgery, and as a form of treatment which may be temporary or, occasionally, permanent in its effect. ${ }^{14}$ BTXA injections were performed as an outpatient procedure, under topical local anaesthetic (amethocaine $1 \%$ ), and carried out under electromyographic control. No injection was given until there was orthoptic evidence that the patient's ocular motility status had remained unchanged for a minimum of 3 months. Two patients underwent strabismus surgery, utilising an adjustable suture technique to ensure optimal ocular alignment. Surgery was performed when orthoptic examination indicated that they had fully recovered from their previous BTXA injection. Details of the 5 patients and their management are summarised below and in Table I.

\section{CASE HISTORIES}

Case 1

A 26-year-old woman presented with an 8 month history of headaches and the recent onset of horizontal diplopia. Systemic prednisolone had produced no significant improvement in her ocular alignment. She had a moderate face turn to the right and a small esophoria with slow recovery. When assessed without her head turn, however, she had a left esotropia ( $45 \Delta$ for near and $35 \Delta$ for distance) and a small left hypotropia with diplopia that could only be abolished in extreme laevoversion (Fig. 1). A CT scan revealed enlargement of the left medial rectus.

Over 6 months there was considerable spontaneous improvement in her field of binocular single vision (Fig. 2). When no further improvement occurred she received a BTXA injection into the right medial rectus. This resulted in centralisation and substantial enlargement of her field of binocular single vision (Fig. 3). She had a small esotropia ( $4 \Delta$ for near, $1 \Delta$ for distance) with a normal head posture and was much more comfortable.

Six months later, however, she suffered a further episode of headache and developed vertical diplopia

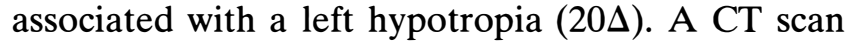
failed to demonstrate involvement of the vertical recti in the inflammatory process. She was treated with ibuprofen and once again there was some improvement, but 18 months later she had persistent diplopia and opted to undergo surgery. This greatly increased her field of binocular single vision (Fig. 4). She has remained asymptomatic and been discharged.

\section{Case 2}

A 37-year-old woman was referred with a history of three episodes of painful diplopia. The first occurred when she was 9 years old. At the age of 24 years she suffered a second episode, involving her right eye,

Table I. Summary of patients: outcome of initial botulinum toxin injections

\begin{tabular}{|c|c|c|c|c|c|c|c|c|c|}
\hline $\begin{array}{l}\text { Patient } \\
\text { no. }\end{array}$ & Age/sex & $\begin{array}{l}\text { Affected } \\
\text { muscles }\end{array}$ & Treatment & $\begin{array}{l}\text { Deviation pre-toxin } \\
\text { (near/distance) }\end{array}$ & $\begin{array}{l}\text { Muscle } \\
\text { injected }\end{array}$ & $\begin{array}{l}\text { Deviation post-toxin } \\
\text { (near/distance) }\end{array}$ & $\begin{array}{l}\text { Total no. } \\
\text { injections }\end{array}$ & $\begin{array}{l}\text { Subsequent } \\
\text { surgery }\end{array}$ & $\begin{array}{c}\text { Final } \\
\text { outcome }\end{array}$ \\
\hline 1 & $25 \mathrm{~F}$ & LMR & $S$ & $\begin{array}{l}30 \Delta \text { ET, } 8 \Delta \text { RHT } \\
30 \Delta \text { ET, } 8 \Delta \text { RHT }\end{array}$ & RMR & $\begin{array}{l}4 \Delta \mathrm{ET}, 2 \Delta \text { RHT } \\
1 \Delta \mathrm{ET}, 2 \Delta \text { RHT }\end{array}$ & 1 & 1 & Asymptomatic \\
\hline 2 & $37 \mathrm{~F}$ & LMR & $\mathbf{N}$ & $\begin{array}{l}35 \Delta \mathrm{ET} \\
35 \Delta \mathrm{ET}\end{array}$ & RMR & $\begin{array}{c}4 \Delta \mathrm{ET}, 3 \Delta \mathrm{RHT} \\
2 \Delta \mathrm{ET}\end{array}$ & 1 & 0 & Asymptomatic \\
\hline 3 & $31 \mathrm{~F}$ & $\begin{array}{l}\text { RLR/RSR } \\
\text { RMR/LLR }\end{array}$ & $\mathrm{N}, \mathrm{S}, \mathrm{R}$ & $\begin{array}{l}85 \Delta \mathrm{XT}, 20 \Delta \mathrm{RHT} \\
60 \Delta \mathrm{XT}, 20 \Delta \mathrm{RHT}\end{array}$ & RLR & $\begin{array}{l}25 \Delta \mathrm{XT}, 20 \mathrm{D} \text { RHT } \\
14 \Delta \mathrm{XT}, 20 \Delta \mathrm{RHT}\end{array}$ & 1 & 2 & $\begin{array}{c}\text { Persistent } \\
\text { diplopia }\end{array}$ \\
\hline 4 & $11 \mathrm{M}$ & LMR & A & $\begin{array}{l}52 \Delta X T \\
45 \Delta X T\end{array}$ & LLR & $\begin{array}{l}4 \Delta \mathrm{XT} \\
2 \Delta \mathrm{XT}\end{array}$ & 1 & 0 & Asymptomatic \\
\hline 5 & $26 \mathrm{~F}$ & $\begin{array}{l}\text { LMR } \\
\text { RMR }\end{array}$ & $\mathbf{N}$ & $\begin{array}{l}6 \Delta \mathrm{ET} \\
10 \Delta \mathrm{ET}\end{array}$ & RMR & $\begin{array}{l}4 \Delta \mathrm{XT} \\
4 \Delta \mathrm{XT}\end{array}$ & 2 & 0 & $\begin{array}{l}\text { Asymptomatic } \\
\text { post-toxin }\end{array}$ \\
\hline
\end{tabular}

M, male; F, female; LMR, left medial rectus; RLR, right lateral rectus; RSR, right superior rectus; A, antibiotics; N, non-steroidal antiinflammatory agents; S, prednisolone orally; R, orbital radiotherapy; $\Delta$, prism dioptres; XT, exotropia; ET, esotropia; RHT, right hypertropia. 


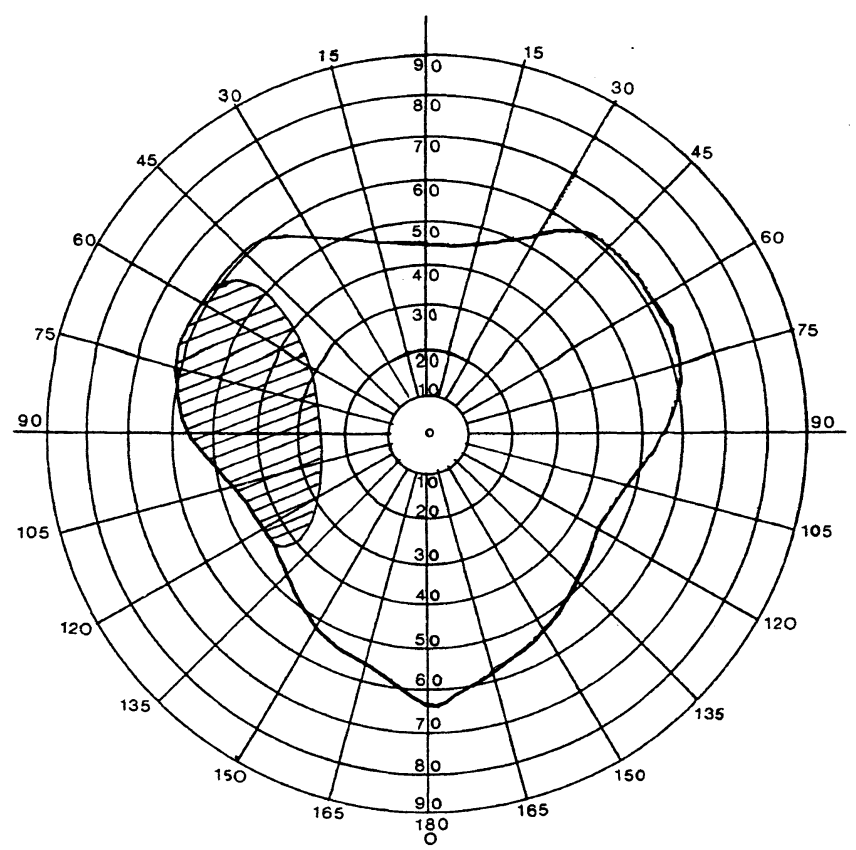

Fig. 1. Case 1. Field of binocular fixation at presentation.

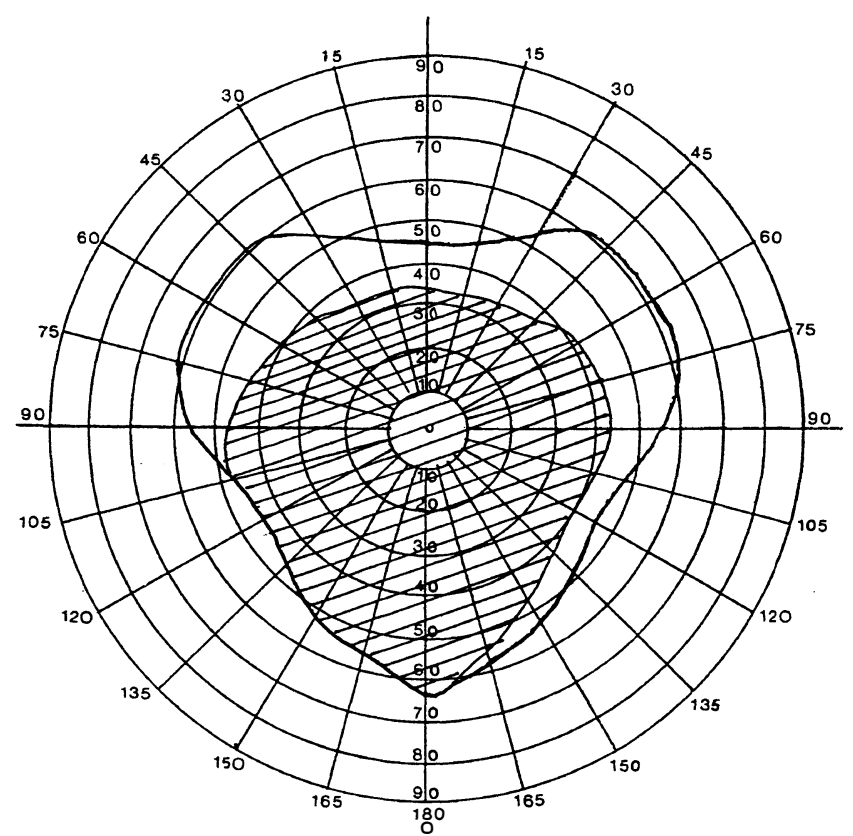

Fig. 3. Case 1. Field of binocular fixation after injection of botulinum toxin $A$ into the right medial rectus muscle.

which resolved spontaneously. The third episode affected her left eye, producing pain, episcleral injection on the medial side of the globe, and an esotropia with horizontal diplopia. A CT scan performed at this time demonstrated enlargement of the left medial rectus. Non-steroidal anti-inflammatory agents relieved her pain, but the diplopia persisted.

She was seen at Moorfields nearly 2 years after this episode and was found to have a persistent $35 \Delta$ right esotropia. She received an injection of BTXA into her right medial rectus. After this she regained

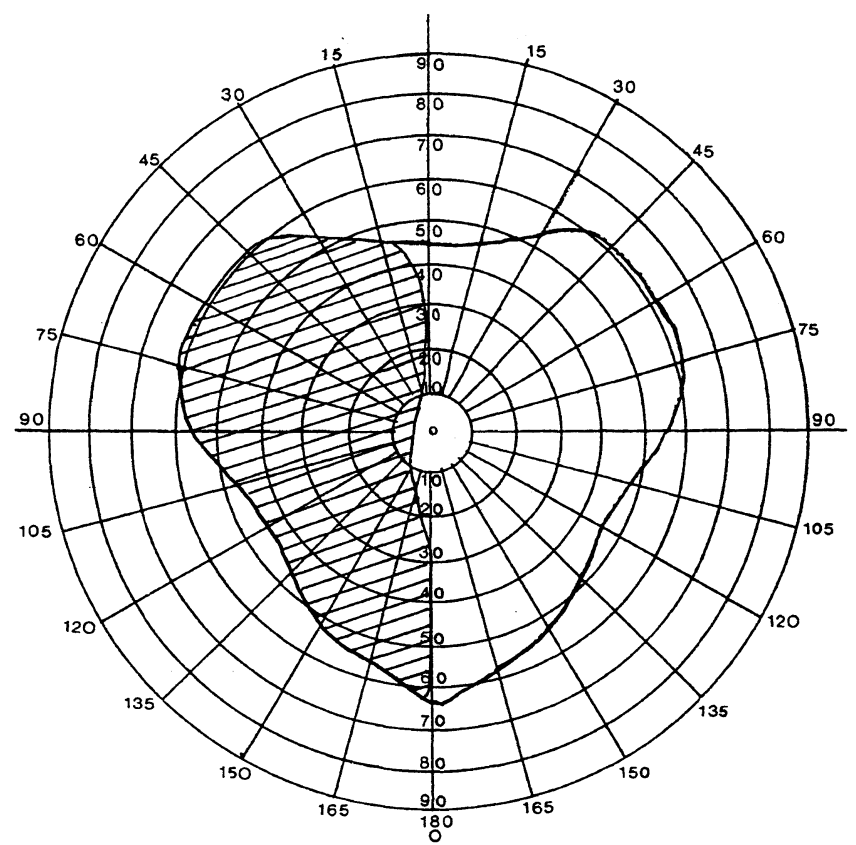

Fig. 2. Case 1. Field of binocular fixation 6 months after presentation.

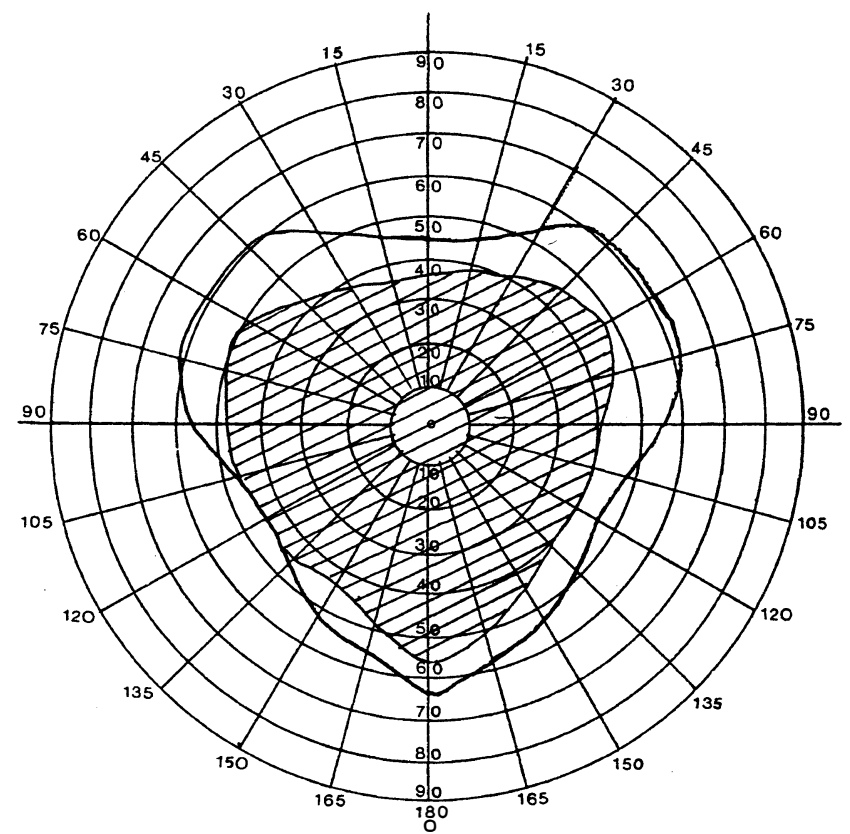

Fig. 4. Case 1. Field of binocular fixation following surgery.

binocular single vision and she was discharged 12 months later with a minimal exophoria.

\section{Case 3}

A 20-year-old woman presented to Moorfields Eye Hospital with a 4 month history of painful ophthalmoplegia affecting the right eye. She had markedly restricted adduction of the right eye, associated with diplopia on laevoversion and retraction of the globe. A CT scan performed at that time revealed enlargement of the right lateral rectus muscle. During the following 12 months she suffered two 
more episodes of myositis resulting in constant diplopia in all positions of gaze. Two further CT scans revealed enlargement of the right medial, lateral and superior recti and of the left lateral rectus. Treatment with oral prednisolone (at a dose of $80 \mathrm{mg} /$ day) was insufficient and she received bilateral orbital radiotherapy $(20 \mathrm{~Gy})$. The involvement of additional muscles increased her exotropia to $85 \Delta$ and resulted in a $20 \Delta$ right hypertropia.

At this point, over a year after the onset of her symptoms, she received a BTXA injection into the right lateral rectus muscle. Electromyography demonstrated definite activity in this muscle. This dramatically reduced her exotropia to $25 \Delta$ for near and $14 \Delta$ for distance, but was not sufficient to overcome her diplopia. After the effects of the toxin injection had worn off she underwent two surgical procedures which produced an enormous improvement in cosmesis, leaving her with a small

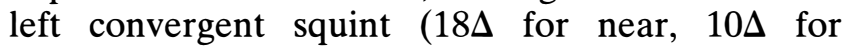

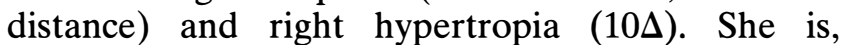
however, still troubled by intractable diplopia and remains unable to return to her work as a beautician.

\section{Case 4}

An 11-year-old boy presented with a 3 week history of a painful left eye, associated with swelling and erythema of the lids and horizontal diplopia. He had a left exotropia measuring $80 \Delta$, with limitation of both abduction and adduction, but no significant proptosis. A CT scan demonstrated isolated enlargement of the left medial rectus without evidence of any other orbital inflammation or of sinusitis. Over a 6 week period the lid swelling subsided and he regained a full range of ocular movements. Despite this an exotropia of $50 \Delta$ remained and his diplopia persisted.

Six months after the onset of his symptoms he received a BTXA injection into his left lateral rectus muscle. After a single injection he regained binocular single vision with 60 seconds of arc stereoacuity (TNO test). He was followed up for 18 months and remained asymptomatic with a small, well-controlled exophoria.

\section{Case 5}

A 24-year-old woman presented with a 1 month history of right eye pain and horizontal diplopia. She had $3 \mathrm{~mm}$ of right proptosis, injection over the medial rectus insertions in both eyes, and a small left esotropia ( $12 \Delta$ for near, $16 \Delta$ for distance). Her field of binocular single vision was very small. A CT scan demonstrated modest enlargement of both medial recti with anterior thickening of the tendons suggestive of myositis. Treatment with oral Froben (flurbiprofen) and oral prednisolone $(80 \mathrm{mg} / \mathrm{day})$ decreased the angle of her esotropia to $6 \Delta$ for near and $10 \Delta$ for distance, but did not abolish her diplopia. Fourteen months later she received a BTXA injection into the right medial rectus. A week after the injection she had a small exophoria, no diplopia in the primary position, and a good field of binocular single vision.

Two years later she began to suffer from diplopia again, and was found to have a small right/alternating esotropia ( $10 \Delta$ for near, $14 \Delta$ for distance). A further BTXA injection was performed, following which she has remained asymptomatic.

\section{RESULTS}

Our 5 patients consisted of 4 women and 1 boy, ranging in age from 11 to 37 years. CT scans revealed involvement of one or both of the medial recti in the inflammatory process in all 5 patients. Three patients had bilateral involvement: 1 simultaneously and symmetrically (both medial recti), and the other 2 sequentially. A vertical deviation was seen in 3 cases, of which 1 was confirmed to be due to involvement of the superior rectus/levator complex by a CT scan. Recurrence of disease occurred in 3 patients, 1 of whom reported suffering three episodes, the first and last of which were separated by 28 years.

In 4 of the 5 patients the limitation of ocular movements was in the field of action of the antagonist of the affected muscle. This pattern is typical of the restrictive motility defect found when a muscle becomes tight secondary to fibrosis. In the remaining patient, who had by far the shortest duration of symptoms, there was limitation of movement in the direction of action of the affected medial rectus.

In 4 patients BTXA injection was successful in eliminating diplopia. In 2 cases a single injection restored binocular single vision and a permanent cure was achieved (there was no recurrence of diplopia or of disease activity at follow-up 12 and 18 months later, respectively). In a third case the patient was asymptomatic following her injection until a recurrence of orbital myositis in another muscle necessitated further treatment. Surgery was then performed which was again successful in abolishing her diplopia. In the fourth case a repeat injection was required after a period of 2 years. Sadly in the remaining patient, although a substantial cosmetic improvement was brought about by surgery, intractable diplopia has persisted.

\section{DISCUSSION}

Following the recent publication of a series of 75 patients with orbital myositis from Miami there are now more than 150 cases reported in the literature. ${ }^{10}$ A small proportion of these patients have been described as subsequently developing signs of underlying systemic disease, but in the great majority the disease remained idiopathic in origin. Almost every series records a female to male ratio of 2 to 1 or 
greater, with only one report from India of a male preponderance. ${ }^{9}$ The majority of patients present for the first time between the ages of 18 and 40 years, but an age range of 9-84 years has been reported. ${ }^{2,3,7,10}$ Four of our 5 subjects were female and all were below the age of 40 years.

We found the medial rectus to be the most commonly involved muscle in our series, in accordance with the majority of published data., ${ }^{2,12}$ In the largest published series, however, involvement of the medial and lateral recti occurred with almost equal frequency. ${ }^{10}$ Of the vertical recti the superior rectus muscle is much more frequently involved in the myositic process than the inferior rectus, in direct contrast to the pattern seen in dysthyroid eye disease (TED). A review of the literature (including our patients) indicates that of 217 muscles affected by orbital myositis the superior rectus was involved in 45 eyes as compared with 18 in which the inferior rectus was involved. Involvement of the oblique muscles is rare. ${ }^{10}$

Both the simultaneous involvement of several extraocular muscles and the recurrence of disease activity are common in orbital myositis. During an acute attack 26 of 75 patients (32\%) were reported to have involvement of more than one muscle. ${ }^{10}$ More significantly up to $80 \%$ of patients may suffer one or more recurrences of their disease, and these recurrences frequently affect different extraocular muscles of the same eye or even muscles of the contralateral eye. ${ }^{3}$ In a series of 15 patients with chronic myositis the majority of cases were found to be bilateral and asymmetrical, with one eye predominantly affected first and then, after an interval, the other eye. ${ }^{7}$ One patient in this series had 12 relapses over a 5 year period. The reported frequency of multiple muscle involvement may, therefore, relate to the duration of disease activity at the time of presentation. In addition the use of sagittal and coronal CT scans by some authors may have resulted in the recognition of muscle enlargement, particularly of the vertical recti, which might have gone undetected with orbital ultrasonography or axial CT scans. ${ }^{3}$

The nature and severity of the ophthalmoplegia in orbital myositis appear also to be related to the duration of disease activity. At the time of diagnosis those patients who present within a week of the onset of symptoms may have normal ocular motility, whilst those presenting at around 2 weeks after onset will tend to have a muscle paresis with decreased ocular movement in the field of action of the affected extraocular muscle. ${ }^{10}$ Those who present later frequently have a restrictive ocular motility defect, similar to that seen in TED, or a mixed paretic and restrictive defect. Since involvement of the lateral recti is common in orbital myositis a restrictive exotropia may be encountered in myositis which would be unusual in TED.

High-dose oral corticosteroids are the treatment of choice in orbital myositis and there is usually a rapid response to this treatment, especially in the acute phase of the disease. There is evidence, from the only two studies which record the incidence of long-term ocular motility defects after an episode of myositis, that those patients who do not receive appropriate therapy in the acute phase are far more likely to suffer persistent ophthalmoplegia. In the first of these studies 5 of the 6 patients who failed to respond to corticosteroids and went on to require either prisms or surgical correction of their strabismus had presented, and received steroid treatment, when they were already in the restrictive phase of their disease. ${ }^{10}$ In the second study 7 of 12 patients presented more than 2 weeks after the onset of their symptoms. Of these 7, 6 had persistent ocular motility defects at the final examination; in contrast, all 5 who received early treatment demonstrated normal ocular motility. ${ }^{3}$ Neither paper comments on the nature of these motility defects or on the manner in which they were managed.

We suggest that a botulinum toxin (BTXA) injection should be the first line of treatment for all patients in whom an ocular motility defect persists after an episode of orbital myositis. In this study BTXA injection was successful in eliminating diplopia in 4 of our 5 patients, and this effect became permanent in 2 cases. Three of these 4 patients were suffering from a restrictive pattern of motility defect and the injection was given into the apparently contracted extraocular muscle. This indicates that BTXA can be used successfully to treat both paretic and restrictive motility defects. Similar results have been obtained in the treatment of the restrictive ophthalmoplegia caused by TED. ${ }^{11}$ In the treatment of a condition such as orbital myositis, in which recurrences are common and may occur years after the initial episode, BTXA may be especially valuable as it can reduce the number of surgical procedures performed upon a given patient. Patient 4 in our series suffered a recurrence of her myositis in a different extraocular muscle several months after a successful BTXA injection and would have required two or more operations if BTXA had not been used. Even where BTXA treatment is not curative it may be used to determine whether a patient has the potential to achieve binocular single vision after a corrective surgical procedure. If there proves to be no potential for single vision the patient can decide whether their diplopia has been made worse by the BTXA injection and if so may decline cosmetic strabismus surgery.

Surgery is indicated in the management of strabismus secondary to orbital myositis when, following a BTXA injection which has produced a satisfactory but temporarv elimination of diplonia. a 
patient opts for a permanent correction of their motility defect. The patient should be counselled regarding the possibility that a recurrence of myositis may necessitate further treatment, and be offered the opportunity to have repeated BTXA injections in place of surgery. We suggest that surgery is carried out utilising an adjustable suture technique because this offers the best prospect of accurate ocular alignment and the elimination of diplopia. In patients with both a horizontal and a vertical component to their motility disorder a combined procedure on the horizontal and a vertical recti may be performed. Since chronic orbital myositis tends to have a restrictive pattern, recession of the extraocular muscles is preferable to resection. If several recurrences occur then repeated BTXA therapy may be indicated rather than multiple surgical procedures.

Lastly we suggest that no treatment, either toxin injection or surgery, should be performed until the patient has remained orthoptically stable for a period of at least 3 months. This allows both for the spontaneous recovery of function which may occur after corticosteroid treatment and for the full development of any restrictive motility defect. Surgery must also be withheld until the effects of a BTXA injection have completely worn off.

Key words: Botulinum toxin, Orbital myositis, Strabismus.

\section{REFERENCES}

1. Kennerdell JS, Dresner SC. The nonspecific orbital inflammatory syndromes. Surv Ophthalmol 1984;29: 93-116.
2. Slavin ML, Glaser JS. Idiopathic orbital myositis: a report of six cases. Arch Ophthalmol 1982;100:1261-5.

3. Weinstein GS, Dresner SC, Slamovits TL, Kennerdell JS. Acute and subacute orbital myositis. Am J Ophthalmol 1983;96:209-17.

4. Blodi FC, Gass JMD. Inflammatory orbital pseudotumour. Br J Ophthalmol 1968;52:79-93.

5. Trokel SL, Hilal SK. Recognition and differential diagnosis of enlarged extraocular muscles in computed tomography. Am J Opthalmol 1979;87:503-12.

6. Dua HS, Smith FW, Singh AK, Forrester JV. Diagnosis of orbital myositis by nuclear magnetic resonance imaging. Br J Ophthalmol 1987;71:54-7.

7. Bullen CL, Young BR. Chronic orbital myositis. Arch. Ophthalmol 1982;100:1749-51.

8. Purcell JJ, Taulbee WA. Orbital myositis after upper respiratory tract infection. Arch Ophthalmol 1981; 99:437-8.

9. Sekhar GC, Mandal AK, Vyas P. Non-specific orbital inflammatory diseases. Doc Ophthalmol 1993;84: 155-70.

10. Siatkowski RM, Capo H, Byrne SF, Gendron EK, Flynn JT, Munoz M, Feuer WJ. Clinical and echographic findings in idiopathic orbital myositis. Am J Ophthalmol 1994;118:343-50.

11. Moorman CM, Elston JS. Acute orbital myositis. Eye 1995;9:96-101.

12. Lenderink T, Jager MJ, Bruijn JA, de Keizer RJ. Immunohistology of eye muscle in idiopathic orbital inflammatory disease (pseudotumor), Graves' ophthalmopathy and healthy controls. Graefes Arch Clin Exp Ophthalmol 1993;231:99-103.

13. Chavis RM, Garner A, Wright JE. Inflammatory orbital pseudotumor: a clinicopathologic study. Arch Ophthalmol 1978;96:1817-22.

14. Lyons CJ, Vickers SF, Lee JP. Botulinum toxin therapy in dysthyroid strabismus. Eye 1990;4:538-42. 